\title{
CORRIGENDUM
}

\section{1 in reflection}

Asher Mullard

Nature Reviews Drug Discovery 11, 6-8 (2012) | doi:10.1038/nrd3643

Daclatasvir was originally misidentified as an NS5A protease inhibitor, when it is an NS5A replication complex inhibitor.

This has been corrected in the online version of the article. 